\title{
Knowledge, Attitudes and Behavior of Bangkok Metropolitan Women Regarding Cervical Cancer Screening
}

\author{
Woraphot Chaowawanit $^{1}$, Siriwan Tangjitgamol ${ }^{1 *}$, Nuttavut Kantathavorn ${ }^{2}$, \\ Natacha Phoolcharoen ${ }^{2}$, Thannaporn Kittisiam ${ }^{1}$, Jakkapan Khunnarong ${ }^{1}$, Busaba \\ Supawattanabodee $^{3}$, Sunamchok Srijaipracharoen ${ }^{1}$, Thaovalai Thavaramara ${ }^{1}$, \\ Kamol Pataradool ${ }^{1}$
}

\begin{abstract}
Purpose: To assess knowledge, attitudes and cervical cancer screening behavior of Bangkok Metropolitan women. Materials and Methods: Thai women, aged 25-to-65 years old, having lived in Bangkok for 5 years or more were invited to participate in the study. After signing informed consent, all women were asked to complete a self-questionnaire (Thai language) with literate assistance if needed. The questionnaire was divided into 3 parts: (I) demographic data; (II) knowledge about cervical cancer screening; and (III) behavior and attitudes, towards cervical cancer screening. Adequate screening was defined as women who had $\geq$ two cervical cancer screening tests except women aged 25-30 years who may have only one screening, and the last screen was within 5 year or had had regular screening. Results: Of 4,339 women, there were $1,857(42.8 \%)$ with adequate screening and $\mathbf{2 , 4 8 2}(\mathbf{5 7 . 2 \%})$ with inadequate screening. Significant factors associated with inadequate screening included age $<$ 45 years, pre-menopausal status, family monthly income $<625$ USD, no reported sexual intercourse, nulliparous, no knowledge, lack of awareness and poor attitudes. Three major reasons provided by women for inadequate screening were no symptoms (54.4\%), fear of pain (33.2\%), and embarrassment (34.6\%). Conclusions: Personal features, knowledge, and attitudes influence screening behavior of Bangkok Metropolitan women. The three most common reasons of women for not undergoinging screening are no symptoms, fear of pain, and embarrassment. These factors should be the focus of attention to improve coverage of cervical cancer screening in Bangkok.
\end{abstract}

Keywords: Knowledge, attitudes - behavior - cervical cancer screening - Bangkok Metropolitan

Asian Pac J Cancer Prev, 17 (3), 945-952

\section{Introduction}

Cervical cancer is the fourth most common cancer in women worldwide with an estimated 528,000 new cases and 266,000 deaths in 2012 (Ferlay et al., 2015). The majority of cervical cancer ( $84 \%$ or 445,000 cases) and deaths $(87 \%$ or 230,000 cases $)$ occurred in less developed regions. This high mortality to incidence ratio (approximately half) in less developed regions was partly because majority of the patients were in locally-advanced stage at diagnosis (Moore et al., 2010). In Thailand, cervical cancer is the second most common cancer among women with annual new cases and deaths from cervical cancer of 8,184 and 4,513, respectively in 2012 (International Agency for Research on Cancer, 2012).

Effective cancer screening is a major factor to reduce cancer incidence and mortality by detecting preinvasive cervical lesions resulting in a high rate of cure after treatment. Several techniques of cervical cancer screening have been used. The most commonly used method nowadays is cytologic test either conventional Papanicolaou (Pap) smear (Papanicolaou and Traut, 1941) or by liquid-based preparation (Arbyn et al., 2008). After a discovery of human papilloma virus (HPV) as the cause of cervical cancer (zurHausen, 1977), HPV DNA testing has become an effective means of screening (Saslow et al., 2012). Another practical test in low resources setting, where cervical cytology of HPV test are inadequate or unavailable, is visual inspection with acetic acid (VIA) (Sankaranarayanan et al., 2003; Gaffikin et al., 2003; Sanghvi et al., 2008; Paul et al., 2013; Bradford and Goodman, 2013). Several countries have set guideline in the screening program including important issues e.g. ages of women, methods, frequency, and etc (Saslow et al., 2012; Massad et al., 2013; National Health Service, 2013). In Thailand, the Ministry of Public Health (MOPH) solicited a cervical cancer screening program for women aged 30-60 years by Pap test every 5 years to 
cover most target population (National Cancer Institute, Thailand, 2013). The Royal Thai College of Obstetrics and Gynaecology (RTCOG) also released a practice guideline of cervical cancer screening for women aged 25-65 years through Pap smear or liquid-based cytology every 2 years and co-test of cytology with HPV DNA testing every 3 years for women aged $>30$ years. The interval may be longer if 3 prior consecutive tests are unremarkable (The Royal Thai College of Obstetrics and Gynaecology, 2012). Of note, only conventional cytologic Pap test is covered by the national health reimbursement system.

Unfortunately, certain numbers of women have never had any or sparingly have screening tests. According to the National Cancer Institute of Thailand, the Pap smear coverage rate during 2006-2009 was only $27.7 \%$ (Khuhaprema et al., 2012). The coverage rate was approximately the same in each year of the study although many strategies from MOPH were used, such as, government funding, continual promotion among health care providers and women in different areas of the country, etc. Although the data of 2005-2009 showed that high-grade squamous intraepithelial lesion (HSIL) and cervical cancer were detected in $0.6 \%$ among screened Thai women (Khuhaprema et al., 2012), the actual prevalence of cervical cancer in Thailand was still as high as 17.8/100,000 (International Agency for Research on Cancer, 2012). This was probably due to the unrevealed HSIL and cervical cancer in the unscreened population.

Focusing on the reasons why women did not have cervical cancer screening, reports from many countries had various findings (Tran et al., 2012; Baskaran et al., 2013; Núñez-Troconis et al., 2013; Cunningham et al., 2015). Several basic characteristics of the women may result in various reasons, such as, education, obstetrics and gynecologic history, and familial or cultural background, etc.Studies from South America, Africa and Asia reported that absence of symptoms, no knowledge, lack of time, embarrassment, fear of pain, anxiety on privacy violation and fear of infection, etc. were obvious reasons for the non-screening. In Thailand, studies from suburb areas of the country reported several reasons for no or suboptimal cervical cancer screening e.g. shyness, no knowledge about the screening, thinking that they had no risks, etc (Kritpetcharat et al., 2003; Budkaew and Chumworathayi, 2014; Wongwatcharanukul et al., 2014).

Bangkok is the capital of the country with the registered population of 5.7 million in 2014 or $8.7 \%$ of the total population within the country (Annual Population Statistics, 2014). Among these, 3 million were female, and 1.8 million were at the age of 25-65 years. Evaluation of the situation in this region is crucial to project a plan our health service for women in this area and the country. Only one previous report from Bangkok identified some problems of screening behavior of health care providers in the hospital (Thanapprapasr et al., 2012). These data may not reflect or represent as the characteristics of women at large in Bangkok. Our hospital is under the administration of the Bangkok Metropolitan area, this study was conducted to assess knowledge, attitude and the cervical cancer screening behavior of Bangkok women in general.

\section{Materials and Methods}

This study was a parallel project to another study for the evaluation on the prevalence of abnormal cervical cytology and HPV DNA among women in Bangkok (in preparation). The study was collaboration between Faculty of Medicine Vajira Hospital, Navamindradhiraj University and Chulabhorn Hospital. Prior to the study, questionnaire to evaluate knowledge, attitudes and behavior of cervical cancer screening of women was contemplated, discussed, tried out in 30 women who sought for medical care in the institution, then adjusted and discussed again until consensus. The final questionnaire was validated by 3 experts (not involved in the study) in the Obstetrics and Gynecology of the institution. The protocol was approved by the Human Research Ethics Committee of both institutions. The study was conducted at both institutions from mid of September until the end of December 2014. Approximately 100 women from each district in Bangkok were planned for an approximate total number of 5,000 women. Thai women aged 25-65 years and had lived in Bangkok for 5 years or over were invited into the study. Exclusion criteria were women with history of: preinvasive or invasive cervical lesions or other gynecologic cancers, hysterectomy for any reasons, HPV vaccination, or being pregnant. Women who could not provide any information were also excluded.

Women were solicited to participate in the study via press conference and mass media including newspaper, television, broadcast, website, posters and brochures. Information sheet about the project was given to all participants who met inclusion criteria. Verbal explanation was also provided in suspicious issues. All women signed their written informed consent and completed a Thai-language questionnaire by themselves with literate assistance if required. Any questions about the questionnaire would be clarified by our research assistants before proceeding. After completing the questionnaire, co-testing by liquid-based cytology and HPV DNA tests was performed. The questionnaire was divided into 3 parts: (I) demographic data including obstetric and gynecologic histories, (II) awareness and behavior of cervical cancer screening, and (III) knowledge and attitudes about cervical cancer screening.

Demographic data included: age, marital status, occupation, education level, religion, family monthly income, smoking history, underlying diseases, menstrual status, sexual activity, number or parity and contraceptive use. Awareness was assessed by 1 first simple question whether the women were aware or knew about cervical cancer screening. Behavior of the screening was evaluated by 3 questions of having 'ever' vs. 'never' had screening, frequency, and the last testing. The women were grouped as 'adequate screening' if they had $\geq$ two cervical cancer screening tests and the last screening was within 5 year or with prior regular screening. An exception was women aged 25-30 years who may have only one screening. The other women were grouped as 'inadequate screening' if they had never had screening, had only one screening, had irregular screening or the last screening was more than 5 years. 
Knowledge was determined with 5 questions about the screening: 1) self-perception regarding knowledge about screening, 2) time to start, 3) frequency, 4) time to stop, and 5) methods. The correct answers in questions numbers 2-4 were based on either the policy of the MOPH or the RTCOG guideline for cervical cancer screening. There were one or more correct items for each question. There must be no false items to consider that answer as correct. Women were categorized into 2 groups for statistical analysis purpose, $0-1$ correct answers were regarded as 'no knowledge' while 2-4 correct answers as 'having knowledge'. Those who had all 4 correct answers were remarked as having good knowledge. The attitudes towards screening were surveyed by 12 possible reasons hindering them from screening: having thought that the test was unnecessary, perception of having no risk for cervical cancer, no symptoms, no time, fear of pain, embarrassment, fear of abnormal results, bad impression with health care services, residing distant from health care unit, concern about the expenses, fear of bleeding or discharge due to vaginal exam, or fear of non-hygienic equipment. Blank spaces were given for additional comments. The women were grouped into 'good attitude' towards the screening unless one or more of these items indicating 'poor attitude' was selected.

Data were analyzed using licensed SPSS statistical software, version 22.0 (IBM Corporation, Armonk, NY, USA). Descriptive statistics were used to analyze demographic data and were summarized as numbers with percentage, mean with standard deviation or median with range. Data between the 'adequate' and 'inadequate' screenings were compared using Chi-square test and student-t test as appropriate.

\section{Results}

There were 5,160 Bangkok women interested to participate in this study. Among these, 755 women did not meet inclusion criteria by various reasons and 66 women without screening history were excluded. A total of 4,339 women were included into the study. Mean age was 46.6 \pm 9.9 years old. The majority $(63.3 \%)$ were at the age of 41-60 years old. Approximately $60 \%$ had education higher than bachelor degree or had family monthly income more than 625 USD (1 USD approximate to $32 \mathrm{Baht}$ ). The two most common occupations were employees (43.9\%) and housewives (26.3\%). Almost all women were Buddhist (94.9\%) and non-smoker (96.9\%). Regarding the obstetric and gynecologic history, slightly more than half of the women $(52.8 \%)$ were pre-menopausal. More than half (63.1\%) were married and 1,077 (24.4\%) were single. Only $412(9.5 \%)$ of women in this study had never had sexual intercourse. Nearly two-third women $(63.0 \%)$ were parous and with history of vaginal delivery $(61.2 \%)$. Characteristic features of women were shown in Table 1.

Out of 4,339 Bangkok women who participated in this study, 3,585 (82.6\%) were aware that cervical cancer could be early detected by screening. However, only 1,144 (26.4\%) had some degrees of knowledge regarding time to start $(1,020$ or $23.5 \%)$, frequency of screening (459 or $10.5 \%$ ), time to stop ( 856 or $19.7 \%$ ), and methods of the screening $(1,938$ or $44.7 \%)$. Of note, only 21 women $(0.5 \%)$ had correct answers in all items.

For the behavior of screening, 2,482 women (57.2\%) were categorized as having inadequate screening for the following reasons: 1,507 women (34.7\%) had never had screening, $798(18.4 \%)$ who aged more than 30 years and had screening only once, and $177(4.1 \%)$ had $\geq 2$ tests but the last screening was $>5$ years. The remaining 1,857 women $(42.8 \%)$ were grouped as having adequate screening. In univariable analysis, we compared the characteristic features of women with adequate and inadequate screening and found factors significantly

Table 1.Characteristics of All Women who Participated in the Study $\left(N=4,339^{a}\right)$

\begin{tabular}{|c|c|}
\hline Demographic characteristics & $\begin{array}{c}\text { Total } \\
\mathrm{N}(\%)\end{array}$ \\
\hline Age group, mean age \pm SD (years) & $46.6 \pm 9.9$ \\
\hline $25-30$ & $299(3.9)$ \\
\hline $31-40$ & $951(21.9)$ \\
\hline $41-50$ & $1,429(32.9)$ \\
\hline $51-60$ & $1,318(30.4)$ \\
\hline $61-65$ & $342(7.9)$ \\
\hline \multicolumn{2}{|l|}{ Menstrual status } \\
\hline Pre-menopause & $2,300(53.0)$ \\
\hline Post-menopause & $2,021(46.6)$ \\
\hline \multicolumn{2}{|l|}{ Education level } \\
\hline No education & $56(1.3)$ \\
\hline Primary education & $502(11.6)$ \\
\hline High school/ Diploma & $1,191(27.4)$ \\
\hline Bachelor & $2,062(47.5)$ \\
\hline Above Master’s degree & $513(11.8)$ \\
\hline \multicolumn{2}{|l|}{ Family monthly income (USD) } \\
\hline$<310$ & $480(11.0)$ \\
\hline $311-625$ & $1,182(27.2)$ \\
\hline $626-940$ & $887(20.4)$ \\
\hline$>940$ & $1,719(39.6)$ \\
\hline \multicolumn{2}{|l|}{ Religion } \\
\hline Buddhist & $4,117(94.9)$ \\
\hline Christian & $92(2.1)$ \\
\hline Muslim & $101(2.3)$ \\
\hline \multicolumn{2}{|l|}{ Occupation } \\
\hline Unemployed / Housewife & $1,143(26.3)$ \\
\hline Employee & $1,906(43.9)$ \\
\hline Personal business & $501(11.5)$ \\
\hline Government officer & $692(15.9)$ \\
\hline Othersb & $19(0.4)$ \\
\hline \multicolumn{2}{|l|}{ Smoking } \\
\hline No & $4,203(96.9)$ \\
\hline Yes & $112(2.6)$ \\
\hline \multicolumn{2}{|l|}{ Underlying disease } \\
\hline No & $3,087(71.1)$ \\
\hline Yes & $1,241(28.6)$ \\
\hline \multicolumn{2}{|l|}{ Sexual intercourse } \\
\hline Never & $411(9.5)$ \\
\hline Ever & $3,911(90.1)$ \\
\hline \multicolumn{2}{|l|}{ Parity } \\
\hline 0 & $1,468(33.8)$ \\
\hline 1 & $964(22.2)$ \\
\hline$>2$ & $1,805(41.6)$ \\
\hline \multicolumn{2}{|l|}{ Contraceptive use } \\
\hline No & $1,246(28.7)$ \\
\hline Yes & 3,077 (70.9) \\
\hline
\end{tabular}

${ }^{a}$ Sixty six women (1.5\%) did not reveal their history of screening; $b$ Other occupations were graduate students, farmers. 
Table 2. Factors contributing to women have inadequate screening $\left(\mathrm{N}=4,339^{\mathrm{a}}\right)$

\begin{tabular}{|c|c|c|c|c|c|c|c|c|c|}
\hline \multirow[t]{2}{*}{ Factors } & Total & \multicolumn{2}{|c|}{ Adequate $=1,857$} & \multicolumn{2}{|c|}{ Inadequate $=2,482$} & \multirow{2}{*}{ 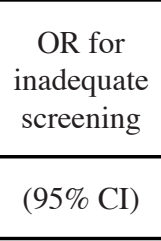 } & \multirow[t]{2}{*}{$\mathrm{p}$-value } & \multirow{2}{*}{$\begin{array}{c}\begin{array}{c}\text { Adjusted } \\
\text { OR for } \\
\text { inadequate } \\
\text { screening }\end{array} \\
(95 \% \mathrm{CI})\end{array}$} & \multirow[t]{2}{*}{ p-value } \\
\hline & $\mathrm{N}(\%)$ & $\mathrm{N}(\%)$ & $\begin{array}{r}\text { Loss } \\
(\%) \\
\end{array}$ & $\mathrm{N}(\%)$ & $\begin{array}{c}\text { Loss } \\
(\%)\end{array}$ & & & & \\
\hline Age & & & & & & & $<0.001$ & & $<0.001$ \\
\hline$<45$ & $\begin{array}{r}1,930 \\
(44.5)\end{array}$ & $\begin{array}{r}729 \\
(39.3)\end{array}$ & $0(0)$ & $\begin{array}{r}1,201 \\
(48.4)\end{array}$ & $0(0)$ & 1.17 & & 1.49 & \\
\hline$>45$ & $\begin{array}{l}2,409 \\
(55.5)\end{array}$ & $\begin{array}{l}1,128 \\
(60.7)\end{array}$ & & $\begin{array}{l}1,281 \\
(51.6)\end{array}$ & & $(1.11-1.23)$ & & $(1.30-1.71)$ & \\
\hline Menstrual status & & & & & & & $<0.001$ & & \\
\hline Pre-menopause & $2,300(53)$ & $\begin{array}{r}923 \\
(49.7)\end{array}$ & $7(0.4)$ & $\begin{array}{l}1,377 \\
(55.5)\end{array}$ & $11(0.4)$ & 1.11 & & & \\
\hline Post-menopause & $\begin{array}{r}2,021 \\
(46.6)\end{array}$ & $\begin{array}{r}927 \\
(49.9)\end{array}$ & & $\begin{array}{r}1,094 \\
(44.1)\end{array}$ & & $(1.05-1.17)$ & & & \\
\hline Education & & & & & & & 0.836 & & \\
\hline Below bachelor & $\begin{array}{r}1,749 \\
(40.3)\end{array}$ & $\begin{array}{r}745 \\
(40.1)\end{array}$ & $7(0.4)$ & $\begin{array}{r}1,004 \\
(40.5)\end{array}$ & $8(0.3)$ & 1 & & & \\
\hline Bachelor or higher & $\begin{array}{l}2,575 \\
(59.3)\end{array}$ & $\begin{array}{l}1,105 \\
(59.5)\end{array}$ & & $\begin{array}{r}1,470 \\
(59.2)\end{array}$ & & $(0.95-1.06)$ & & & \\
\hline \multicolumn{2}{|c|}{ Family monthly income (USD) } & & & & & & $<0.001$ & & $<0.001$ \\
\hline$<625$ & $\begin{array}{r}1,662 \\
(38.3)\end{array}$ & $\begin{array}{r}644 \\
(34.7)\end{array}$ & $\begin{array}{r}24 \\
(1.3)\end{array}$ & $\begin{array}{r}1,010 \\
(40.7)\end{array}$ & $55(2.2)$ & 1.12 & & 1.32 & \\
\hline$>625$ & $\begin{array}{l}2,606 \\
(60.1)\end{array}$ & $\begin{array}{l}1,189 \\
(64.0)\end{array}$ & & $\begin{array}{l}1,417 \\
(57.1)\end{array}$ & & $(1.07-1.18)$ & & $(1.15-1.51)$ & \\
\hline Religion & & & & & & & 0.202 & & \\
\hline Buddhist & $\begin{array}{l}4,117 \\
(94.9)\end{array}$ & $\begin{array}{l}1,752 \\
(94.3)\end{array}$ & $\begin{array}{r}14 \\
(0.8)\end{array}$ & $\begin{array}{l}2,365 \\
(95.3)\end{array}$ & $15(0.6)$ & 1.09 & & & \\
\hline Other & $193(4.4)$ & $91(4.9)$ & & $102(4.1)$ & & $(0.95-1.25)$ & & & \\
\hline Sexual intercourse & & & & & & & $<0.001$ & & $<0.001$ \\
\hline Never & $411(9.5)$ & $89(4.8)$ & $5(0.3)$ & $\begin{array}{r}322 \\
(13.0)\end{array}$ & $12(0.5)$ & 1.43 & & 1.66 & \\
\hline Ever & $\begin{array}{r}3,911 \\
(90.1)\end{array}$ & $\begin{array}{l}1,763 \\
(94.9)\end{array}$ & & $\begin{array}{l}2,148 \\
(86.5)\end{array}$ & & $(1.35-1.51)$ & & $(1.25-2.20)$ & \\
\hline Parity & & & & & & & $<0.001$ & & $<0.001$ \\
\hline Nulliparous & $\begin{array}{r}1,468 \\
(33.8)\end{array}$ & $\begin{array}{r}445 \\
(24.0)\end{array}$ & $\begin{array}{r}26 \\
(1.4)\end{array}$ & $\begin{array}{r}1,023 \\
(41.2)\end{array}$ & $76(3.1)$ & 1.39 & & 1.92 & \\
\hline Multiparous & $\begin{array}{r}2,769 \\
(63.8)\end{array}$ & $\begin{array}{l}1,386 \\
(74.6)\end{array}$ & & $\begin{array}{r}1,383 \\
(55.7)\end{array}$ & & $(1.33-1.47)$ & & $(1.63-2.25)$ & \\
\hline \multicolumn{3}{|c|}{ Knowledge about cervical cancer screening } & & & & & 0.004 & & $<0.001$ \\
\hline No knowledge & $\begin{array}{l}3,194 \\
(73.6)\end{array}$ & $\begin{array}{r}1,326 \\
(71.4)\end{array}$ & $0(0)$ & $\begin{array}{r}1,868 \\
(75.3)\end{array}$ & $1(0)$ & 1.09 & & 1.21 & \\
\hline Have knowledge & $\begin{array}{r}1,144 \\
(26.4)\end{array}$ & $\begin{array}{r}531 \\
(28.6)\end{array}$ & & $\begin{array}{r}613 \\
(24.7)\end{array}$ & & $(1.03-1.16)$ & & $(1.04-1.4)$ & \\
\hline Awareness & & & & & & & $<0.001$ & & $<0.001$ \\
\hline No & $739(17.0)$ & $\begin{array}{r}179 \\
(9.6)\end{array}$ & $\begin{array}{r}10 \\
(0.5)\end{array}$ & $\begin{array}{r}560 \\
(22.6)\end{array}$ & $5(0.2)$ & 1.42 & & 3.63 & \\
\hline Yes & $\begin{array}{l}3,585 \\
(82.6)\end{array}$ & $\begin{array}{r}1,668 \\
(89.8)\end{array}$ & & $\begin{array}{r}1,917 \\
(77.2)\end{array}$ & & $(1.35-1.49)$ & & $(2.95-4.45)$ & \\
\hline Attitude & & & & & & & $<0.001$ & & $<0.001$ \\
\hline Poor attitude & $\begin{array}{l}3,603 \\
(83.0)\end{array}$ & $\begin{array}{r}1,405 \\
(75.8)\end{array}$ & $7(0.4)$ & $\begin{array}{l}2,198 \\
(88.6)\end{array}$ & $5(0.2)$ & 1.58 & & 3.03 & \\
\hline Good attitude & 724 (16.7) & $\begin{array}{r}445 \\
(24.0) \\
\end{array}$ & & $\begin{array}{r}279 \\
(11.2) \\
\end{array}$ & & $(1.44-1.74)$ & & $(2.51-3.65)$ & \\
\hline
\end{tabular}

${ }^{a}$ Sixty six women (1.5\%) did not reveal their history of screening; OR, odd ratio; CI, confident interval; Factors significant from univariable analyses were entered for multivariable analyses except menopausal status

younger age was also a significant factor associated with inadequate screening (Claeys et al., 2002; Thanapprapasr et al., 2012). For women who had low income, they may focus on economical issues than to maintain healthy 
Table 3. Attitudes or reasons why Women have Inadequate Cervical Cancer Screening $\left(N=4,339^{a}\right)$

\begin{tabular}{|c|c|c|c|}
\hline Attitudes or reasons of having or not having screening test $\mathrm{t}^{\mathrm{b}}$ & Adequate $=1,857^{\mathrm{c}}$ & Inadequate $=2,482^{\mathrm{c}}$ & p-value \\
\hline & $\mathrm{N}(\%)$ & $\mathrm{N}(\%)$ & \\
\hline \multicolumn{4}{|l|}{ Self-perception } \\
\hline Unnecessary & $34(1.8)$ & $90(3.6)$ & $<0.001$ \\
\hline No risks of cervical cancer & $210(11.3)$ & $406(16.4)$ & $<0.001$ \\
\hline No symptoms & $781(42.1)$ & $1,347(54.3)$ & $<0.001$ \\
\hline No time to get the screening & $397(21.4)$ & $492(19.8)$ & 0.189 \\
\hline Fear of pain & $425(22.9)$ & $823(33.2)$ & $<0.001$ \\
\hline Embarrassment & $459(24.7)$ & $858(34.6)$ & $<0.001$ \\
\hline Fear of abnormal results & $204(11.0)$ & $424(17.1)$ & $<0.001$ \\
\hline \multicolumn{4}{|l|}{ Health care provider } \\
\hline Bad impression with health services & $204(11.0)$ & $287(11.6)$ & 0.579 \\
\hline Far from health care services & $53(2.9)$ & $77(3.1)$ & 0.649 \\
\hline \multicolumn{4}{|l|}{ Procedure } \\
\hline Expensive cost of cervical cancer screening & $209(11.3)$ & $469(18.9)$ & $<0.001$ \\
\hline Fear of bleeding or discharge due to vaginal exam & $64(3.4)$ & $111(4.5)$ & 0.093 \\
\hline Fear of non-hygienic equipment & $193(10.4)$ & $372(15.0)$ & $<0.001$ \\
\hline
\end{tabular}

a Sixty six women $(1.5 \%)$ did not reveal their history of screening; ${ }^{b}$ more than one answer was allowed; ${ }^{c}$ Loss data : adequate group $=10(0.5 \%)$ and inadequate group $=5(0.2 \%)$

associated with inadequate screening were: aged $<45$ years, pre-menopause, family monthly income $<625$ USD, no sexual intercourse, nulliparous, no knowledge about cervical cancer screening, unawareness and poor attitudes (Table 2). These factors were also significant in multivariable analysis.

Attitudes towards the screening or reasons the women did not have screening were explored. There were no significant differences on the following items between those with adequate and inadequate screening: no time, bad impression with health care providers and services, residing distant from health care units, fear of bleeding or discharge due to vaginal exam. Women in the inadequate group reported significantly higher frequency of the followings: no necessity, no risks of cervical cancer, no symptoms, fear of pain, embarrassment, fear of abnormal results, concerning on the cost of screening, and fear of non-hygienic equipments. The three most common reasons reported by the women to explain inadequate screening were: no symptoms $(1,347$ or $54.3 \%)$, fear of pain (823 or $33.2 \%$ ), and embarrassment (858 or $34.6 \%$ ). (Table 3).

\section{Discussion}

As mentioned earlier that this study was a parallel project with the co-test screening of cytology and HPV DNA test in Bangkok women. We excluded the women with history of pre-invasive or invasive cervical lesions or other gynecologic cancers or even HPV vaccination, so the knowledge and attitudes of these women should be different from normal population. Women who had hysterectomy for any reasons were not included because they might have already had some information about cervical cancer screening. Furthermore, there were no indications for further screening in these women.

Our study demonstrated that majority $(83 \%)$ of Bangkok women were aware of cervical cancer screening.
However, only $26 \%$ had knowledge and only $0.5 \%$ had 'good knowledge' with all correct answers. Compared to previous study from one suburb area of the country, only $14 \%$ of the women had knowledge about the screening (Budkaew and Chumworathayi, 2014). One factor might be the level of education. Nearly $60 \%$ of women in our study had bachelor degree or higher of education compared to $40 \%$ in that study.

Despite being in a capital city where lots of development like health services should be optimal, $35 \%$ of the women had never had a cervical cancer screening at all. The 'never had' screening rate in our study was much higher than $15 \%$ (more than 2 folds) (Charakorn et al., 2011) but much lower than 62\% (half) (Thanapprapasr et al., 2012) in two previous reports from one hospital in Bangkok. Reports from suburb area of Thailand found $33 \%$ or $35 \%$ of 'never had' screening (Kritpetcharat et al., 2003; Wongwatcharanukul et al., 2014). In capital cities of other countries, the rates of 'no screening' varied widely from 15-90\% (Claeys et al., 2002; Al Sairafi and Mohamed, 2009; Baskaran et al., 2013; Núñez-Troconis et al., 2013; Cunningham et al., 2015). Aside from the $35 \%$ of 'never had' screening, $18 \%$ and $4 \%$ of Bangkok women had only one test or the last test was more than 5 years, making up $57 \%$ of the inadequate screening in our study. Another study from suburb area of Thailand reported 68\% of the women had the last screening more than 5 years or inadequate screening by our definition (Budkaew and Chumworathayi, 2014).

The causes of different rates of 'no' or 'inadequate' screening among studies in Thailand and across the countries may vary, such as, cultural background, education level, or economics, etc.Significant characteristics associated with inadequate screening in our study were $<45$ years, pre-menopause, family monthly income $<625$ USD, no sexual intercourse, and nulliparous. Younger women were less likely to have health problems, so with less concern about their health. In previous studies, 
Table 4. Comparison of Previous Studies and our Study in Attitudes and Behavior Towards Cervical Cancer Screening

\begin{tabular}{|c|c|c|c|c|c|}
\hline Study & Participants & $\mathrm{N}$ & $\begin{array}{l}\text { Never } \\
\text { had } \\
\text { screening } \\
\quad(\%)\end{array}$ & $\begin{array}{l}\text { Factors contributing to } \\
\text { women have never had } \\
\text { screening }\end{array}$ & $\begin{array}{l}\text { Attitudes or reasons why women } \\
\text { have never had screening }(\%)\end{array}$ \\
\hline Thanaprapasr et al. & $\begin{array}{l}\text { Health care } \\
\text { providers } \\
\text { Bangkok, } \\
\text { Thailand }\end{array}$ & 1,348 & 62 & $\begin{array}{l}\text { - Age }<30 \text { years } \\
\text { - Unmarried status }\end{array}$ & $\begin{array}{l}\text { - No risk (27) } \\
\text { - Unnecessary (19) } \\
\text { - Embarrassment (15) } \\
\text { - No time (10) } \\
\text { - No symptoms (10) }\end{array}$ \\
\hline Charakorn et al & $\begin{array}{l}\text { Women visiting } \\
\text { gynecology OPD } \\
\text { Bangkok, Thailand }\end{array}$ & 764 & 15 & N/A & N/A \\
\hline Kritpetcharat et al. & $\begin{array}{l}\text { Suburb women } \\
\text { KhonKaen, } \\
\text { Thailand }\end{array}$ & 1,199 & 33 & N/A & $\begin{array}{l}\text { - Embarrassment (42) } \\
\text { - No knowledge (17) } \\
\text { - Fear (11) } \\
\text { - No concern (11) } \\
\text { - No money (9) }\end{array}$ \\
\hline Budkaew et al. & $\begin{array}{l}\text { Suburb women } \\
\text { KhonKaen, } \\
\text { Thailand }\end{array}$ & 195 & $68^{a}$ & $\begin{array}{l}\text { - Monthly family income } \\
<470 \text { USD } \\
\text { - No sexual intercourse } \\
\text { - No knowledge and poor } \\
\text { attitude }\end{array}$ & $\begin{array}{l}\text { - No symptom }(23) \\
\text { - No children }(8) \\
\text { - Unmarried status }(8) \\
\text { - No time }(5) \\
\text { - Financial problem }(2)\end{array}$ \\
\hline $\begin{array}{l}\text { Wongwatcharanukul } \\
\text { et al. }\end{array}$ & $\begin{array}{l}\text { Hill tribe women } \\
\text { Phetchabun, } \\
\text { Thailand }\end{array}$ & 547 & 35 & $\begin{array}{l}\text { - No education } \\
\text { - Animistic beliefs } \\
\text { - Never had pregnancy } \\
\text { - No knowledge about } \\
\text { cervical cancer }\end{array}$ & $\begin{array}{l}\text { - No time (61) } \\
\text { - Embarrassment (44) } \\
\text { - Fear of pain }(21) \\
\text { - No symptoms }(9) \\
\text { - Lack of convenient transport (7) }\end{array}$ \\
\hline Baskaran et al. & $\begin{array}{l}\text { Women visiting } \\
\text { gynecology OPD } \\
\text { Kuala Lumper, } \\
\text { Malaysia }\end{array}$ & 369 & 24.4 & - Low education level & $\begin{array}{l}\text { - No information on procedure } \\
(80) \\
\text { - Embarrassment (70) } \\
\text { - Lack of convenient time (58) } \\
\text { - Feel worried (47) } \\
\text { - Attitudes of health workers (47) }\end{array}$ \\
\hline Tran et al. & $\begin{array}{l}\text { Urban and rural } \\
\text { women } \\
\text { Korea }\end{array}$ & 200 & 94 & N/A & $\begin{array}{l}\text { - Lack of awareness }(48 \%) \\
\text { - Dislike pelvic exam }(47 \%) \\
\text { - No symptoms } \\
(17 \% \text { rural, } 31 \% \text { urban, } \mathrm{p}<0.05) \\
\text { - Far from service (Rural only - } \\
62 \%)\end{array}$ \\
\hline Cunningham et al. & $\begin{array}{l}\text { Urban vs. Rural } \\
\text { women } \\
\text { Kilimanjaro region, } \\
\text { Tanzania }\end{array}$ & $\begin{array}{l}\text { Rural } \\
303 \\
\text { Urban } \\
272\end{array}$ & $\begin{array}{c}94 \\
\text { Rural } 96 \\
\text { Urban } 92\end{array}$ & N/A & $\begin{array}{l}\text { - Unawareness (67) } \\
\text { - No money (49) } \\
\text { - No time }(26) \\
\text { - Far from service }(20) \\
\text { - Not having companion }(7)\end{array}$ \\
\hline $\begin{array}{l}\text { Nunaz-Troconis } \\
\text { et al. }\end{array}$ & $\begin{array}{l}\text { Women visiting } \\
\text { OPD } \\
\text { Maracaibo, } \\
\text { Venezuela }\end{array}$ & 590 & 15 & N/A & $\begin{array}{l}\text { - No knowledge (35) } \\
\text { - Embarrassment (34) } \\
\text { - Fear pain/ something bad (32) } \\
\text { - No physicians explanation (32) }\end{array}$ \\
\hline Claeys et al. & $\begin{array}{l}\text { General population } \\
\text { Rivas, Nigaragua }\end{array}$ & 634 & 42 & $\begin{array}{l}\text { - Age }>45 \text { years } \\
\text { - Low education level } \\
\text { - No knowledge about } \\
\text { cervical cancer }\end{array}$ & N/A \\
\hline Al Sairafi et al & $\begin{array}{l}\text { General population } \\
\text { Kuwait }\end{array}$ & 281 & 65 & $\begin{array}{l}\text {-Younger age } \\
\text {-Younger age at marriage }\end{array}$ & $\begin{array}{l}\text { - Doctor never suggested (48) } \\
\text { - Pain (18) } \\
\text { - Fear of infection (15) } \\
\text { - Embarrassment (13) } \\
\text { - No symptoms (10) }\end{array}$ \\
\hline Our study & $\begin{array}{l}\text { General population } \\
\text { Bangkok, Thailand }\end{array}$ & 4,339 & 35 & $\begin{array}{l}\text { - Age }<45 \text { years } \\
\text { - Pre-menopause } \\
\text { - Monthly family income } \\
<625 \text { USD } \\
\text { - No sexual intercourse } \\
\text { - Nulliparous } \\
\text { - No knowledge/ aware- } \\
\text { ness and poor attitude }\end{array}$ & $\begin{array}{l}\text { - No symptoms }(54) \\
\text { - Embarrassment }(35) \\
\text { - Fear of pain (33) } \\
\text { - No time }(20) \\
\text { - Expensive cost ofcervical cancer } \\
\text { (19) }\end{array}$ \\
\hline
\end{tabular}

${ }^{a}$ Not having been screened within the past 5 years; OPD, outpatient department; N/A, not available 
status, similar to a study from suburb area (Budkaew and Chumworathayi, 2014). A previous study among medical personnel in Bangkok showed that being single was a significant factor for the unscreened women (Thanapprapasr et al., 2012). Our study focused on sexual activity rather than marital status because of modern or Western lifestyles in a metropolis that women are sexually active without marriage. On the other hand, factors influencing screening behavior might be different in other areas. The study of Wongwatcharanukul et al. which focused in hill tribe women in rural area reported no education, never had pregnancy, animistic belief (without religion), and no knowledge as important factors for never screening behavior (Wongwatcharanukul et al., 2014). We would like to emphasize that the underlying factors of 'no' or 'inadequate' screening were rather multi-factorial.In Bangkok, our study which included general population in Bangkok (59\% high education level) revealed that $35 \%$ had never had screening. This was different from two previous studies of Charakorn et al. and Thanapprapasr et al. The study of Charakorn et al. which involved women seeking medical service in the hospital (64\% high education level) showed only $15 \%$ had no screening (Charakorn et al., 2011) while Thanapprapasr study demonstrated that $62 \%$ of healthcare providers in their hospital had never had screening (Thanapprapasr et al., 2012). We proposed that other factors, such as 'concerning' or 'negligence' or 'overconfidence' of the women themselves may contribute to the screening behavior. As found in Thanapprapasr et al. and our studies, $16 \%$ to $27 \%$ of women reported that they had no risks so the test was unnecessary (Thanapprapasr et al., 2012).

Aside from the women's personal features, we found 'no awareness' and 'poor attitude' were significantly associated with the inadequate screening. Similar reasons were reported from several studies; however, with different frequency. Table 4 shows summarized findings of attitudes from various reports.

Limitations of this study were recall bias, unverified data due to self-answer questionnaires. Furthermore, these women were not a real community-based population because they had some concern about their health and actively participated in our project. These factors may affect the knowledge and attitude evaluated.

Our study identified some problems that policy planning should focus on. Knowledge and attitudes towards cervical cancer screening could be improved by various strategies, e.g. health education, public relation or press conferences with mass media through celebrities/ ambassadors or society volunteers to re-assure those women that the screening may cause some discomforts but could be painless without any pelvic pathology, and solicit screening. Health care providers should also ascertain privacy when doing screening and creates a friendly environment to decrease embarrassment. In Thailand where conservative culture is common, self-collected specimen for HPV testing may play an important role. Most of all, all parties involved in the screening should work in collaboration continually until cervical cancer incidence can be reduced.

In conclusion, Despite being in the capital city of
Thailand, approximately one third of the women never had cervical cancer screening. Almost $74 \%$ of them had no knowledge about cervical cancer screening while $83 \%$ had poor attitudes towards screening. The three most common reasons of women for not having screening included: no symptoms, fear of pain, and embarrassment. These factors as well as other negative impression should be addressed by health care providers to improve the coverage rate of cervical cancer screening in Bangkok Metropolis.

\section{Acknowledgements}

The authors are thankful to Miss Siriporn Saeloo, Chulabhorn Hospital, Bangkok, Thailand for facilitation of the data collection.

\section{References}

Al Sairafi M, Mohamed FA (2009). Knowledge, attitudes, and practice related to cervical cancer screening among Kuwaiti women. Med Princ Pract, 18, 35-42.

Annual Population Statistics (2014). Report, The Bureau of Registration Administration, Thailand.

Arbyn M, Bergeron C, Klinkhamer P, et al (2008). Liquid compared with conventional cervical cytology: a systematic review and meta-analysis. Obstet Gynecol, 111, 167-77.

Baskaran P, Subramanian P, Rahman RA, et al (2013). Perceived susceptibility, and cervical cancer screening benefits and barriers in Malaysian women visiting outpatient clinics. Asian Pac J Cancer Prev, 14, 7693-9.

Bradford L, Goodman A (2013). Cervical cancer screening and prevention in low-resourcesettings. Clin Obstet Gynecol, 56, 76-87.

Budkaew J, Chumworathayi B (2014). Factors associated with decisions to attend cervical cancer screening among women aged 30-60 years in Chatapadung Contracting Medical Unit, Thailand. Asian Pac J Cancer Prev, 15, 4903-7.

Charakorn C, Rattanasiri S, Lertkhachonsuk AA, et al (2011). Knowledge of Pap smear, HPV and the HPV vaccine and the acceptability of the HPV vaccine by Thai women. Asia Pac J Clin Oncol, 7, 160-7.

Claeys P, Gonzalez C, Gonzalez M, et al (2002). Determinants of cervical cancer screening in a poor area: results of a population-based survey in Rivas, Nicaragua. Trop Med Int Health, 7, 935-41.

Cunningham MS, Skrastins E, Fitzpatrick R, et al (2015). Cervical cancer screening and HPV vaccine acceptability among rural and urban women in Kilimanjaro Region, Tanzania. BMJ Open, 5.

International Agency for Research on Cancer (2012). Population fact sheets [Internet].

Ferlay J, Soerjomataram I, Ervik M, et al (2015). Cancer incidence and mortality worldwide: sources, methods and major patterns in GLOBOCAN 2012. Int J Cancer, 136, 359-86.

Gaffikin L, Blumenthal PD, Emerson M, Limpaphayom K; Royal Thai College of Obstetricians and Gynaecologists (RTCOG)/ JHPIEGO Corporation Cervical Cancer Prevention Group (2003). Safety, acceptability, and feasibility of a single-visit approach to cervical-cancer prevention in rural Thailand: a demonstration project. Lancet, 361, 814-20.

Khuhaprema T, Attasara P, Srivatanakul P, et al (2012). Organization and evolution of organized cervical cytology screening in Thailand. Int J Gynaecol Obstet, 118, 107-11.

Kritpetcharat O, Suwanrungruang K, Sriamporn S et al (2003). 
The coverage of cervical cancer screening inKhonKaen, Northeast Thailand. Asian Pac J Cancer Prev, 4, 103-5.

Massad LS, Einstein MH, Huh WK, et al (2013). 2012 updated consensus guidelines for the management of abnormal cervical cancer screening tests and cancer precursors. J Low Genit Tract Dis, 17, 1-27.

Moore MA, Attasara P, Khuhaprema T, et al (2010). Cancer epidemiology in mainland South-East Asia - past, present and future. Asian Pac J Cancer Prev, 11, 67-80.

National Cancer Institute, Department of medical services, Ministry of Public Health, Thailand (2013). Guideline for cervical cancer screening [Internet].

National Health Service (2013). Cervical Screening Programme [Internet].

Nunez-Troconis J, Tulliani E, Gabriela Martinez M, Fernandez $\mathrm{N}$ (2013). Knowledge and attitudes as predictors of cervical cancer screening among women in a Venezuelan urban area. Invest Clin, 54, 20-33.

Papanicolaou GN, Traut HF (1941). The diagnostic value of vaginal smears in carcinoma of the uterus. Am J Obstet Gynecol, 42, 193-205.

Paul P, Winkler JL, Bartolini RM, et al (2013). Screen-andtreat approach to cervical cancer prevention using visual inspection with acetic acid and cryotherapy: experiences, perceptions, and beliefs from demonstration projects in Peru, Uganda, and Vietnam. Oncologist, 18, 6-12.

Sanghvi H, Limpaphayom KK, Plotkin M, et al (2008). Cervical cancer screening using visual inspection with acetic acid: operational experiences from Ghana and Thailand. Reprod Health Matters, 16, 67-77.

Sankaranarayanan R, Wesley R, Thara S, et al (2003). Test characteristics of visual inspection with $4 \%$ acetic acid (VIA) and Lugol's iodine (VILI) in cervical cancer screening in Kerala, India. Int J Cancer, 106, 404-8.

Saslow D, Solomon D, Lawson HW, et al (2012). American cancer society, american society for colposcopy and cervical pathology, and american society for clinical pathology screening guidelines for the prevention and early detection of cervical cancer. Am J ClinPathol, 137, 516-42.

Thanapprapasr D, Deesamer S, Sujintawong S, et al (2012). Cervical cancer screening behaviours among Thai women: results from a cross-sectional survey of 2112 healthcare providers at Ramathibodi Hospital, Thailand. Eur J Cancer Care, 21, 542-7.

The Royal Thai College of Obstetrics and Gynaecology (2012). RTCOG guideline for cervical cancer screening [Internet].

Tran NT, Choe SI, Taylor R, et al (2011). Knowledge, attitude and practice (KAP) concerning cervical cancer and screening among rural and urban women in six provinces of the democratic people's republic of Korea. Asian Pac J Cancer Prev, 2, 3029-33.

Wongwatcharanukul L, Promthet S, Bradshaw P, et al (2014). Factors affecting cervical cancer screening uptake by Hmong hilltribe women in Thailand. Asian Pac J Cancer Prev, 15, 3753-6.

zur Hausen H (1977). Human papillomaviruses and their possible role in squamous cell carcinomas. Curr Top Microbiol Immunol, 78, 1-30. 